\title{
PROGNOSTIC FACTORS AND PATHOPHYSIOLOGY OF DIABETES REMISSION AFTER METABOLIC GASTRIC BYPASS, SLEEVE GASTRECTOMY AND GREATER CURVATURE PLICATION: A RANDOMIZED CONTROLLED TRIAL
}

Vilarrasa Núria ${ }^{1,5}$, Casajoana Anna ${ }^{2}$, García Ruiz de Gordejuela Amador ${ }^{2}$, Pujol Jordi², Duran Xavier ${ }^{3,5}$, Garrido Pilar ${ }^{4}$, Monseny Rosa ${ }^{4}$, Vendrell Joan ${ }^{3,5}$, Fernandez Veledo Sonia ${ }^{3,5}$.

${ }^{1}$ Endocrinology Unit. Bellvitge University Hospital-IDIBELL, Barcelona, Spain2'General Surgery and Obesity Unit, Bellvitge University Hospital-IDIBELL, Barcelona, Spain

${ }^{3}$ Hospital Universitari de Tarragona Joan XXIII, Institut Investigació Sanitaria Pere Virgili, Universitat Rovira i Virgili

${ }^{4}$ Clinical Nutrition Unit. Bellvitge's University Hospital-IDIBELL, Barcelona, Spain

${ }^{5}$ CIBER de Diabetes y Enfermedades Metabólicas Asociadas (CIBERDEM), Bellvitge University Hospital-IDIBELL

This work was funded by grant "Ajuts per a projectes de recerca clínica de l'Hospital Universitari de Bellvitge (2011-PR143/11)" and grant FIS Instituto Carlos III (PI 11/01960)

\section{INTRODUCTION}

Thirty-nine percent of morbidly obese patients have type 2 diabetes mellitus (T2DM) and $80 \%$ of them can achieve diabetes remission after bariatric surgery. However, there are few randomized studies comparing the metabolic results of different surgical techniques and the hormonal mechanisms involved. There are several factors related to diabetes improvement after bariatric surgery (BS), among which outstands weight loss (WL), decreased caloric intake, intestinal malabsorption, changes in biliopancreatic salts, and in gut hormones, particularly those implicated in the incretin axis. The influence of each of these factors might vary with the surgical technique and their respective anatomical modifications. By-passing the duodenum provokes an increase in GLP-1 that has been associated to glucose improvement and gastric fundus exclusion causes a reduction in the secretion of the Ghrelin orexigen hormone.

Metabolic Gastric Bypas (mGBP)
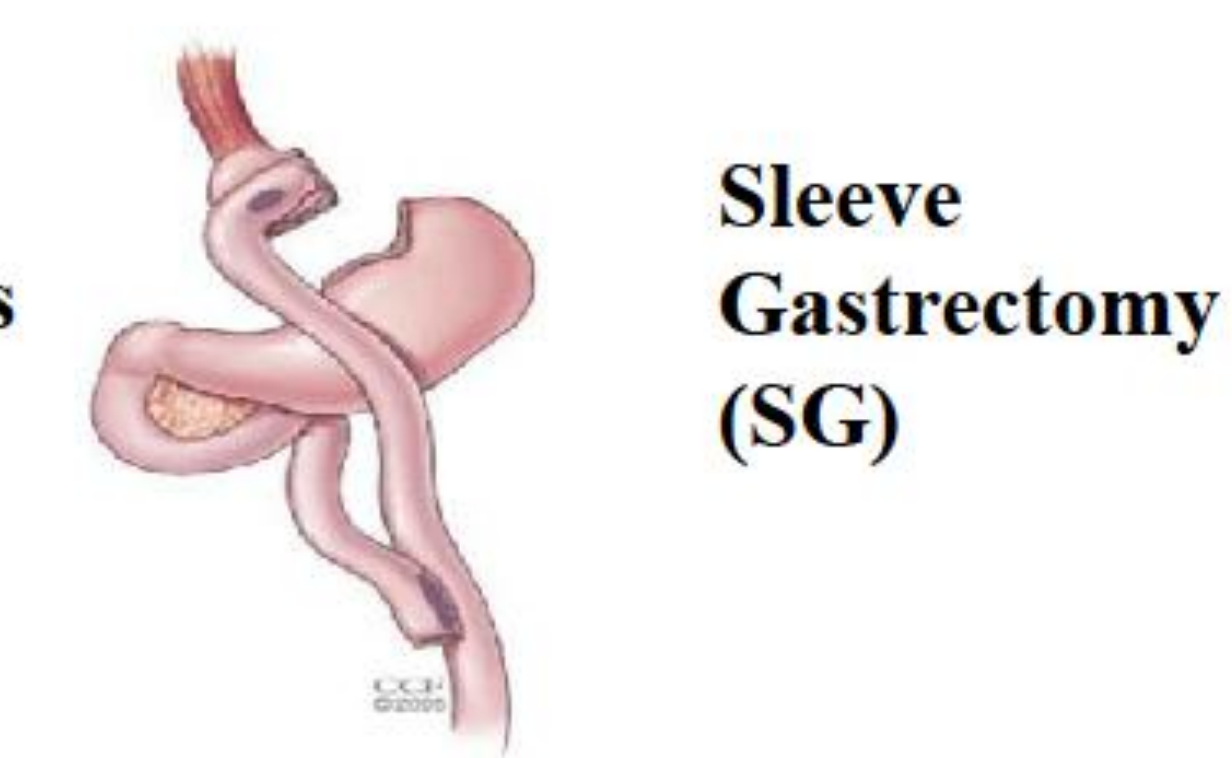
(SG)
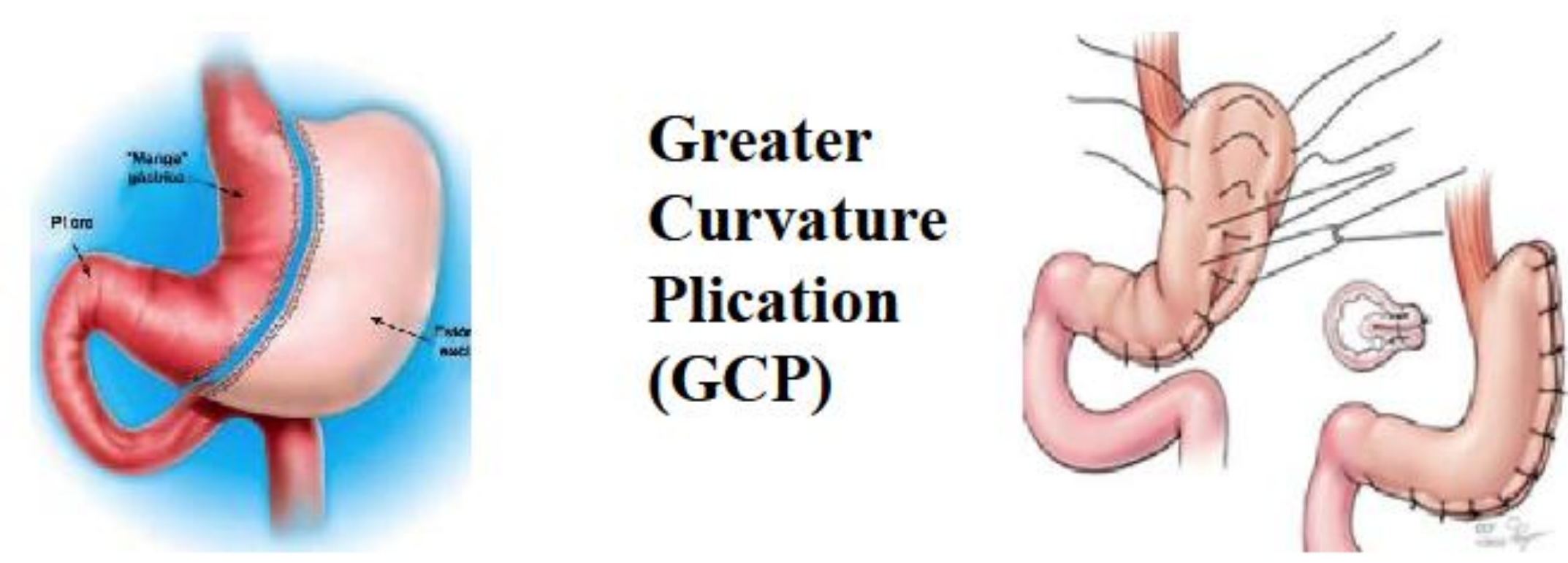

\section{RESULTS}

\begin{abstract}
$\checkmark$ After bariatric surgery (BS), total weight loss was higher at 24 and 48 weeks in mGBP compared with SG and GCP (Figure 1). $\mathrm{HbA}_{1 \mathrm{c}}$ was significantly lower in $\mathrm{mGBP}$ at 12, 24 and 48 weeks compared with SG and GCP (figure 2).

$\checkmark$ AUC for plasma glucose was significantly lower in mGBP at one and 12 months (figure 3). AUC for GLP-1 and GLP-2 increased significantly at one and 12 months after mGBP (figures 4-5); at 12 month fasting concentrations of PYY and ghrelin increased after mGBP (figures 6, 8).
\end{abstract}

\begin{tabular}{|c|c|c|c|c|}
\hline & Total remission & $\begin{array}{l}\text { Partial } \\
\text { remission }\end{array}$ & Non-remission & $\mathrm{p}$ \\
\hline $\operatorname{MGBP}(n, \%)$ & $12(80 \%)$ & $3(20 \%)$ & $0(0,0 \%)$ & \multirow[t]{3}{*}{$<0.00$} \\
\hline SG $(n, \%)$ & $8(53.3 \%)$ & $1(6.67 \%)$ & $6(40 \%)$ & \\
\hline $\operatorname{GCP}(n, \%)$ & $3(20 \%)$ & $4(26.7 \%)$ & $8(53.3 \%)$ & \\
\hline $\begin{array}{l}\sum_{<6}^{\text {Basal }} \mathrm{HbA}_{1 \mathrm{c}}(\%) \\
\text { (\%) }\end{array}$ & $5(83.3 \%)$ & $0(0 \%)$ & $1(16.7 \%)$ & \multirow[t]{3}{*}{$<0.00$} \\
\hline $6-6,5$ & $8(88.9 \%)$ & $1(11.1 \%)$ & $0(0 \%)$ & \\
\hline$>6.5$ & $10(33.3 \%)$ & $7(23.3 \%)$ & $13(43.3 \%)$ & \\
\hline $\begin{array}{l}\text { Diabetes duration } \\
\text { (month) }\end{array}$ & 77 [43.5-114] & $47[31.2-66.5]$ & 160 [105-230] & 0,004 \\
\hline C-Peptide ${ }_{b(n g / m l)}$ & $1.56[1.32-2.13]$ & $1.92[1.80-2.40]$ & $2.10[1.53-2.61]$ & 0.472 \\
\hline $\begin{array}{l}\text { Insulin treatment } \\
\text { Yes }\end{array}$ & $4(23.5 \%)$ & $3(17.6 \%)$ & $10(58.8 \%)$ & \multirow[t]{2}{*}{0,003} \\
\hline Not & $19(67.9 \%)$ & $5(17.9 \%)$ & $4(14.3 \%)$ & \\
\hline Insulin (UI/Kg/day) & $0.52[0.17-0.92]$ & $0.73[0.42-0.74]$ & $0.95[0.67-1.16]$ & 0.115 \\
\hline$W_{b-12 \text { month }}$ & $\begin{array}{c}-30.10 \\
{[-36.42 ;-25.84]}\end{array}$ & $\begin{array}{c}-31.75 \\
{[-33.35 ;-22.08]}\end{array}$ & $\begin{array}{c}-22.00 \\
{[-26.80:-17.30]}\end{array}$ & 0.007 \\
\hline AUC GLP-1 ${ }_{1 \mathrm{~m}-\mathrm{b}}$ & $140[43.7 ; 214]$ & $23.3[-7.80 ; 75.7]$ & $7.24[-31.8 ; 40.2]$ & 0.001 \\
\hline AUC GLP- ${ }_{1 \mathrm{~m}-\mathrm{b}}$ & $67.3[24.8 ; 118]$ & $19.5[8.47 ; 39.9]$ & $23.1[7.26 ; 40.0]$ & 0.009 \\
\hline
\end{tabular}

\section{OBJECTIVES}

To study and compare the improvement of T2DM and the hormonal pathways following three surgical techniques: metabolic gastric bypass (mGBP), sleeve gastrectomy (SG) and greater curvature plication (GCP).

\section{METHODS}

Prospective, randomized controlled single-center study in patients with T2DM and morbid obesity. 45 patients were randomly assigned (1:1:1) to the 3 surgical techniques. (Trial registration ISRCTN 14104758). At baseline, one and 12 months a standard meal test was performed to determine GLP-1, GLP-2, PYY, Ghrelin and glucagon concentrations. GLP-1 was measured by radioimmunoassay (Millipore, Sain Charles, MO). Glucagon and PYY by enzyme immunoassay (Yanahaira Institute Inc., Awakura, Japan). Plasma ghrelin wa determined by enzyme immunoassay (CUSABIO, Wuhan Hi-tech Medical Devices, China).

Table1.- Initial Characteristics of patients

\begin{tabular}{|c|c|c|c|c|}
\hline & mGBP $(n=15)$ & $\mathbf{S G}(\mathrm{n}=15)$ & GCP $(n=15)$ & $p$ \\
\hline Sex H/D & $7 / 8$ & $5 / 10$ & $3 / 12$ & 0.301 \\
\hline Age (year) & $51.10 \pm 7.70$ & $49.20 \pm 9.16$ & $49.70 \pm 8.12$ & 0.827 \\
\hline Weight (Kg) & $103 \pm 10.8$ & $102 \pm 10.8$ & $105 \pm 12$ & 0.907 \\
\hline BMI $\left(\mathrm{Kg} / \mathrm{m}^{2}\right)$ & $38.7 \pm 2.01$ & $39.0 \pm 1.68$ & $40.7 \pm 1.34$ & 0.010 \\
\hline Waist circumference (cm) & $118.87 \pm 7.58$ & $117.53 \pm 7.56$ & $117.33 \pm 8.95$ & 0.882 \\
\hline Total adipose tissue (\%) & $37.58 \pm 7.80$ & $37.91 \pm 5.05$ & $36.36 \pm 11.91$ & 0.989 \\
\hline T2DM duration (months) & $54[39.5 ; 114]$ & $120[51.2 ; 180]$ & $83[45 ; 144]$ & 0.216 \\
\hline C- Peptide (ng/ml) & $3.04 \pm 1.01$ & $3.13 \pm 1.80$ & $3.75 \pm 2.75$ & 0.566 \\
\hline Fasting glucose $(\mathrm{mmol} / \mathrm{L})$ & $8.38 \pm 3,00$ & $9.55 \pm 3.57$ & $9.84 \pm 5,25$ & 0.566 \\
\hline HbA1c (\%) & $7.39 \pm 1.95$ & $7.89 \pm 1.71$ & $8.05 \pm 2.15$ & 0.498 \\
\hline \multicolumn{5}{|l|}{ T2DM treatment [\%, (n)] } \\
\hline Metformin & $100(\mathrm{n}=15)$ & $93.3(\mathrm{n}=14)$ & $80(\mathrm{n}=12)$ & 0.302 \\
\hline Sulfunilurea & $20(\mathrm{n}=3)$ & $13.3(\mathrm{n}=2)$ & $40(\mathrm{n}=6)$ & 0.311 \\
\hline GLP-1 analog & $20(\mathrm{n}=3)$ & $20(\mathrm{n}=3)$ & $26.70(\mathrm{n}=4)$ & 1.000 \\
\hline DPP-IV inhibitor & $26.70(\mathrm{n}=4)$ & $40(\mathrm{n}=6)$ & $6.67(\mathrm{n}=1)$ & 0.130 \\
\hline Insulin treatment [\%, (n)] & $33.30(\mathrm{n}=5)$ & $40(\mathrm{n}=6)$ & $40(\mathrm{n}=6)$ & 0.910 \\
\hline Insulin total dose (UI/day) & $73.20[21-102]$ & $86.67[16-142]$ & $79.71[10-164]$ & 0.835 \\
\hline Insulin dose/Kg/day & $0.72 \pm 0.34$ & $0.84 \pm 0.45$ & $0.78 \pm 0.60$ & 0.751 \\
\hline Dyslipidemia $(\%, \mathbf{n})$ & $73.3(\mathrm{n}=11)$ & $80(\mathrm{n}=12)$ & $86.7(\mathrm{n}=13)$ & 0.894 \\
\hline High blood pressure $(\%, n)$ & $66.7(\mathrm{n}=10)$ & $73.3(\mathrm{n}=11)$ & $80(\mathrm{n}=12)$ & 0.912 \\
\hline
\end{tabular}

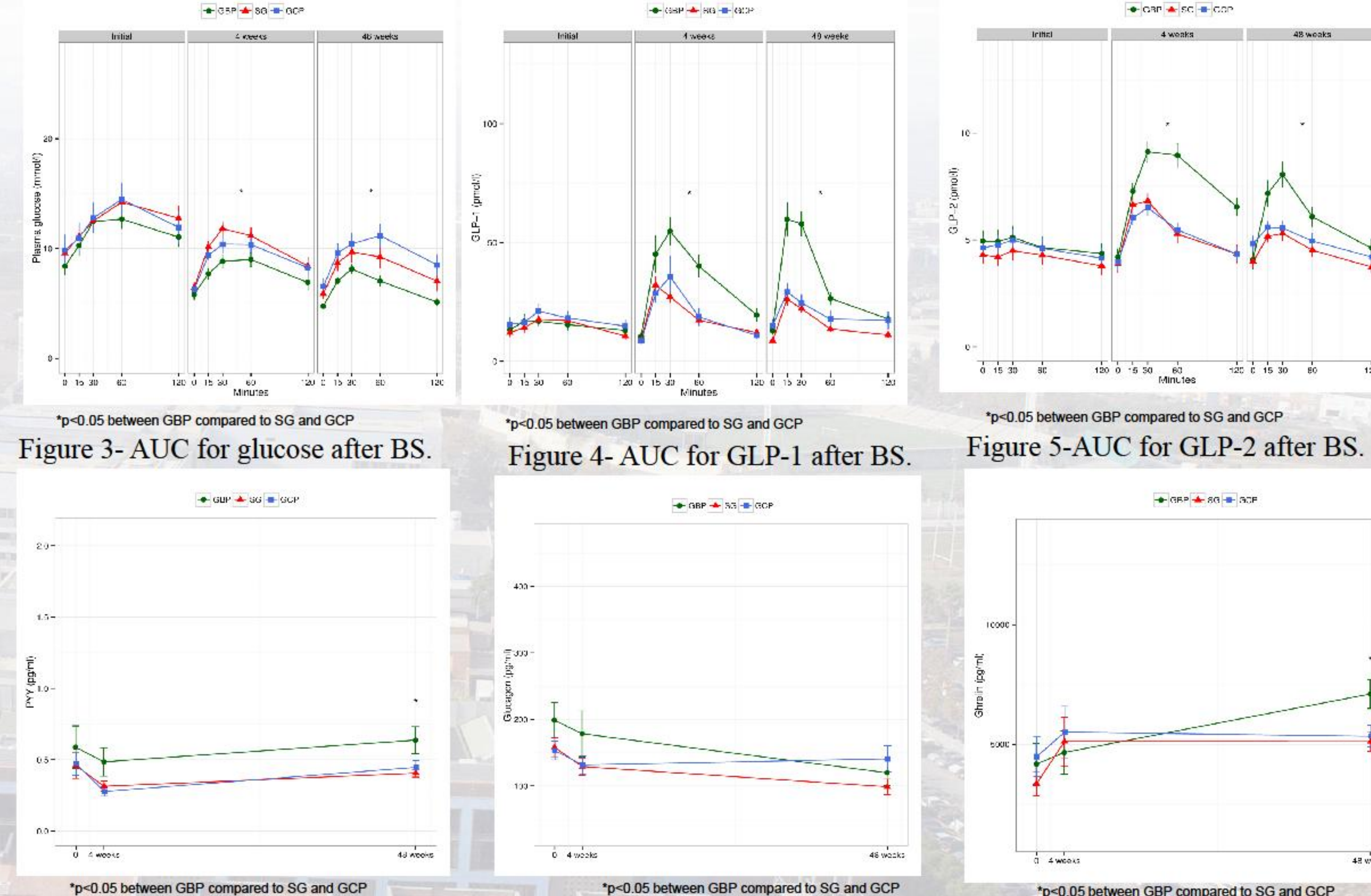

Figure 6 - PYY concentrations after BS. Figure 7- Glucagon con concentrations after BS. Figure 8- Ghrelin concentrations after BS Multiple logistic regression analysis showed a negative association between probability of T2DM remission and insulin treatment ( $B-3.67$, OR $0.025, p=0.018$ ), and a positive association with higher increase in $\triangle A U C$ GLP-1 at month one after BS ( $B 0.021$, OD 1.021, $p=0.013$ ).

No correlation was found between changes in Ghrelin, PYY and glucagon or WL and DM remission.

\section{CONCLUSIONS}

$\checkmark \mathrm{mGBP}$ is the technique that has shown a higher rate of weight loss and T2DM remission compared to SG and GCP.

$\checkmark$ Factors associated with improved glycemic control are those that accompany a less evolved diabetes being the most important the absence of insulin treatment.

$\checkmark$ Regarding hormonal pathways, an enhanced secretion of GLP-1 was associated with a higher rate of T2DM remission

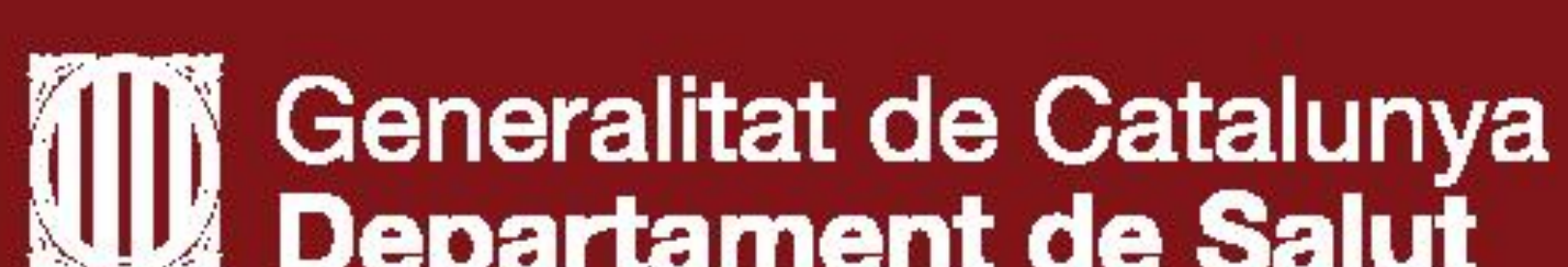

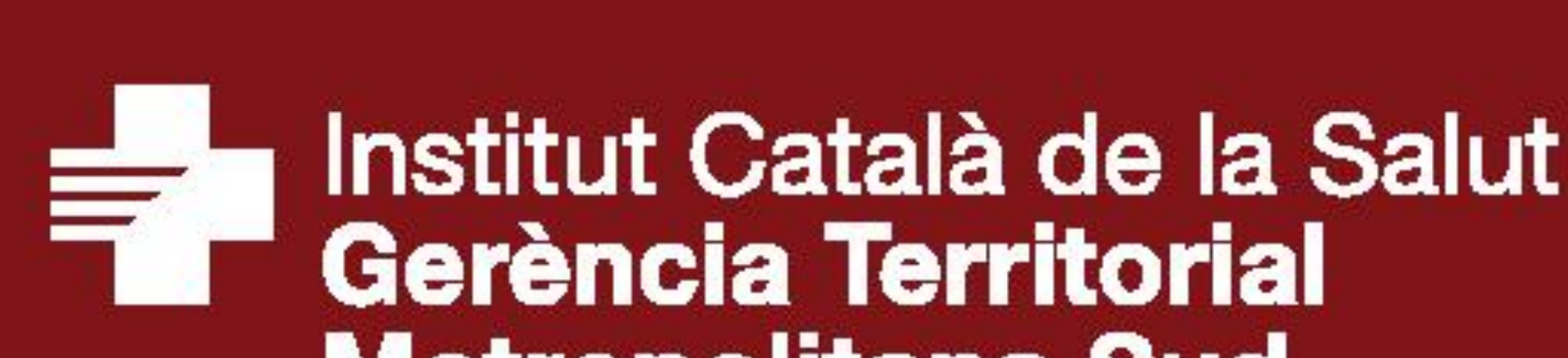
Metropoltana Sud

\section{I|l Bellvitge \\ 1 L Hospital Universitar \\ IDIBELLiq}

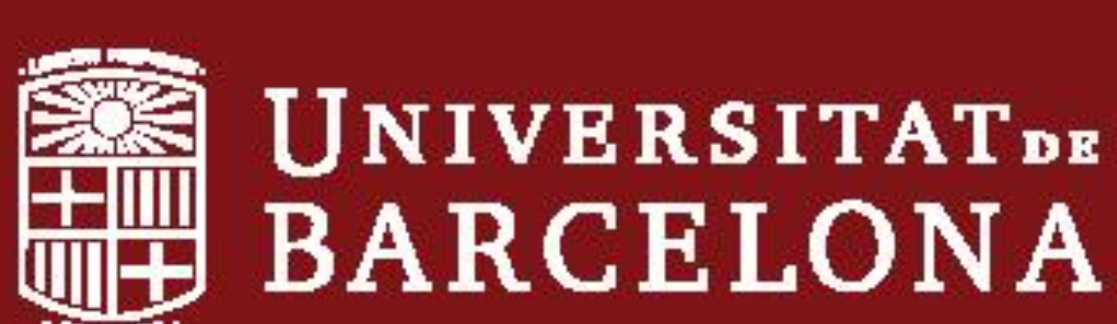

